\title{
Modified Positron Annihilation Model for Glassy-Like $\mathrm{As}_{2} \mathrm{Se}_{3}$
}

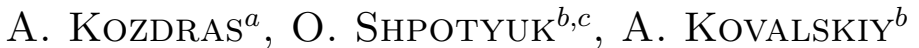 \\ AND J. FILIPECKI ${ }^{c}$ \\ ${ }^{a}$ Physics Laboratory, Opole Technical University \\ Ozimska 75, 45-370 Opole, Poland \\ ${ }^{b}$ Institute of Materials, Scientific Research Company "Carat" \\ Stryjska 202, 79031 Lviv, Ukraine \\ ${ }^{c}$ Institute of Physics, Jan Dlugosz University \\ Al. Armii Krajowej 13/15, 42-201 Czestochowa, Poland
}

\begin{abstract}
An approach to structural characterization of chalcogenide glasses based on the study of void distribution is discussed. The results of positron annihilation lifetime spectra measurements for glassy-like $\mathrm{g}-\mathrm{As}_{2} \mathrm{Se}_{3}$ are compared with nano-void distribution data obtained from Monte Carlo simulation. In this consideration perspectives to involve the parameters of nano-voids calculated from the first sharp diffraction peak in the framework of known Elliott's model are analyzed.
\end{abstract}

PACS numbers: 61.80.Ed, 61.82.Ms, 78.70.Bj

\section{Introduction}

Positron annihilation lifetime spectroscopy (PALS) is rarely used for characterizing vitreous chalcogenide $(\mathrm{ChG})$ semiconductors. This is due to enormous difficulties in interpreting caused by occurrence of long lifetime components in the experimental positron lifetime spectra. A lack of correlation between parameters of annihilation and those of other structurally sensitive methods is an additional obstacle to overcome in order to apply this method for ChG. Systematization of the experimental data available in the literature reveals great differences from study to study in the values of annihilation parameters for ChG having identical chemical composition. The glassy-like $\mathrm{g}-\mathrm{As}_{2} \mathrm{Se}_{3}$ can serve as a typical example [1]. Thus, a need arises to find a model that would address all these major experimental problems beyond any doubt.

To date, two independent approaches to the positron trapping processes in ChG have been developed. In the 80's of the last century, Shantarovich $[2,3]$ presented a hypothesis that electronegatively charged point-like defects within 
vitreous lattice (so-called coordination topological defects) are responsible for positron trapping in ChG. A contrary approach was taken by Jensen et al. [4] in the mid 90's. The theoretical calculations of positron lifetimes for different kinds of vacancy-type defects in crystalline (orthorhombic) c- $\mathrm{As}_{2} \mathrm{Se}_{3}$ were performed on the basis of Puska's positron trapping model [5]. It was assumed that not native point-like charged defects, but only extended vacancy-type voids of 25-100 $\AA^{3}$ free volumes were responsible for positron trapping in both c- $\mathrm{As}_{2} \mathrm{Se}_{3}$ and $\mathrm{g}-\mathrm{As}_{2} \mathrm{Se}_{3}$. The numerical values of positron lifetimes $\tau$ (in ns) were found to be in a linear dependence on free volume $V$ (in $\AA^{3}$ ):

$$
\tau \cong 0.240+0.0013 \mathrm{~V} \text {. }
$$

Taking a critical attitude towards previous results concerning positron lifetimes in $\mathrm{g}-\mathrm{As}_{2} \mathrm{Se}_{3}$, we decided to conduct our own measurements in the hope of being able to identify a trapping concept that could properly describe annihilation in this glass.

\section{Experimental}

Two identical g- $\mathrm{As}_{2} \mathrm{Se}_{3}$ samples prepared from high-purity elemental constituents by direct synthesis in evacuated quartz ampoules were used in our experiment. The standard rocking furnace technique (the highest temperature was $850^{\circ} \mathrm{C}$ ) followed by water quenching was applied [6]. After synthesis, all ingots were cut into plates about $\sim 1.5 \mathrm{~mm}$ in thick and polished to an optical quality.

The experimental PAL measurements were performed with an ORTEC spectrometer $(\mathrm{FWHM}=270 \mathrm{ps})$ using ${ }^{22} \mathrm{Na}(\sim 0.74 \mathrm{MBq})$ source placed between two sandwiched g- $\mathrm{As}_{2} \mathrm{Se}_{3}$ samples.

The obtained spectra (each including $>1 \times 10^{6}$ counts) were mathematically treated with LT computer program [7], the best results corresponding to two-component fitting procedure. Only results with FIT (statistically weighted least-squares deviation between experimental points and theoretical curve) values, which were quite close to 1.0 (the optimal FIT deviation range - from 0.95 up to $\sim 1.1-1.2$ ), were left for the further treatment. Assuming that two-state positron trapping model $[8,9]$ is valid for $\mathrm{g}_{-} \mathrm{As}_{2} \mathrm{Se}_{3}$, the numerical parameters of this model were calculated using $\tau_{1}, \tau_{2}, I_{1}$, and $I_{2}$ values obtained with an applied fitting procedure. The next parameters of the assumed trapping model (bulk positron lifetime $\tau_{\mathrm{B}}$, average positron lifetime $\bar{\tau}$, and positron trapping rate $\kappa_{d}$ ) were calculated, too.

\section{Results and discussion}

Describing positron annihilation in $\mathrm{g}_{-} \mathrm{As}_{2} \mathrm{Se}_{3}$ samples within two-state positron trapping model [8], we select the optimal sets of two-component fitting parameters calculated with LT computer program. Approximately with the 
same probability, the next two groups of parameters are proper to the examined $\mathrm{g}-\mathrm{As}_{2} \mathrm{Se}_{3}$ :

1. $\tau_{1}=0.20 \mathrm{~ns} ; \tau_{2}=0.37 \mathrm{~ns} ; I_{2}=0.60 ; \bar{\tau}=0.30 \mathrm{~ns} ; \tau_{\mathrm{B}}=0.28 \mathrm{~ns} ;$ $\kappa_{d}=1.4 \mathrm{~ns}^{-1}$ and

2. $\tau_{1}=0.22 \mathrm{~ns} ; \tau_{2}=0.37 \mathrm{~ns} ; I_{2}=0.54 ; \bar{\tau}=0.30 \mathrm{~ns} ; \tau_{\mathrm{B}}=0.28 \mathrm{~ns} ;$ $\kappa_{d}=1.0 \mathrm{~ns}^{-1}$.

Explanation of the above experimental PALS results can be done using a nano-void topology of a layer-biased structural model of $\mathrm{g}-\mathrm{As}_{2} \mathrm{Se}_{3}$ containing 146 atoms [10] (Fig. 1). This model includes at least three types of free-volume nano-voids centered near $\bar{r}_{1} \approx 1.5 \AA, \bar{r}_{2} \approx 2.3 \AA$, and $\bar{r}_{3} \approx 2.9 \AA$. These nano-voids can be effective traps for positrons with characteristic lifetimes in dependence on their individual trapping rates. Consequently, two separate components in the experimentally measured PAL spectra may differ sufficiently for different combinations of individual free-volume nano-voids. In accordance to Jensen's approach calculations (Eq. (1)), the numerical value of long lifetime, associated with the greatest nano-voids centered near $\bar{\tau}_{3} \approx 2.9 \AA$, corresponds exactly to $\tau_{2}=0.37 \mathrm{~ns}$ in full agreement with the obtained experimental results. This testifies that the values of positron trapping rate $\kappa_{d}$ for another two types of nano-voids (components $\bar{r}_{1} \approx 1.5 \AA$ and $\bar{r}_{2} \approx 2.3 \AA$ ) are sufficiently low. Within a two-state model, these nano-voids contribute into $\tau_{1}$ lifetime. A three-component (or even more) model is ineffective for interpretation of experimental PALS results, because of high FIT and limited resolution of the PALS method.

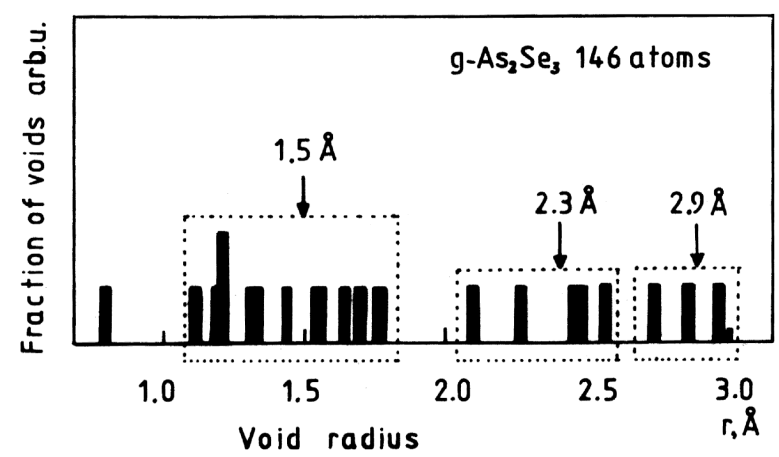

Fig. 1. Nano-void radius distribution for layer-biased $\mathrm{g}-\mathrm{As}_{2} \mathrm{Se}_{3}$, obtained by Monte Carlo simulations (after data from [10]).

Elliott [11] has proposed a new explanation for structural origin of the first sharp diffraction peak (FSDP), which is a characteristic feature in the measured structure factor of many disordered solids, through a void-based model. The application of Elliott's model of the FSDP in order to estimate the volume in interstitial nano-voids around cation-centered units is limited in the case of $\mathrm{g}-\mathrm{As}_{2} \mathrm{Se}_{3}$. The 
reason is that the analytical relation connecting the FSDP position, $Q_{\mathrm{FSDP}}$, and free-volume nano-void diameter, $d$

$$
Q_{\mathrm{FSDP}}=3 \pi / 2 d=1.5 \pi / d \approx 4.7 / d
$$

has been derived only for monoatomic tetravalent $\mathrm{AX}_{2}$ glasses, but not for g- $\mathrm{As}_{2} \mathrm{Se}_{3}$. This relation was firstly introduced by Bletry [12] in the framework of closed-packed random network of aggregating particles. This approach is not valid for $\mathrm{g}-\mathrm{As}_{2} \mathrm{Se}_{3}$, having pyramidal $\mathrm{As}\left(\mathrm{Se}_{1 / 2}\right)_{3}$ units of principally another topological nature in its structural network. However, by accepting that the FSDP origin in $\mathrm{g}-\mathrm{As}_{2} \mathrm{Se}_{3}$ and $\mathrm{g}-\mathrm{AX}_{2}$ is the same, the real relation between $Q_{\mathrm{FSDP}}$ and $d$ values can be established empirically, taking into account the results obtained with the PALS method. Indeed, the nano-void volume attributed to defect-related $\tau_{2}=0.37$ ns lifetime component can be estimated as $\sim 100 \AA^{3}$, which corresponds to a nano-void radius of $\sim 2.9 \AA$. If the same type of free-volume nano-voids is responsible for FSDP data, giving $Q_{\mathrm{FSDP}} \approx 1.2 \AA^{-1}[1,4]$, then instead of formula (2) the following equation should be used:

$$
Q_{\mathrm{FSDP}} \approx 2.2 \pi / d \text {. }
$$

Thus, in the case of $\mathrm{g}-\mathrm{As}_{2} \mathrm{Se}_{3}, Q_{\mathrm{FSDP}}$ and $d$ are connected through a coefficient quite close to $2 \pi$ that is a typical situation for XRD patterns obtained from crystallographically-ordered atomic planes. It probably means that the FSDP-related free-volume nano-voids originate from interlayer separation regions in a glass network.

\section{Conclusion}

PALS provides an elegant experimental probe of free-volume distribution and, along with Monte Carlo simulation and FSDP techniques, can be used to characterize nano-void topology in network glasses such as g- $\mathrm{As}_{2} \mathrm{Se}_{3}$.

\section{References}

[1] O.I. Shpotyuk, A.P. Kovalskiy, J. Filipecki, M. Hyla, A. Kozdras, Physica B 342344, 960 (2003).

[2] B.V. Kobrin, V.P. Shantarovich, Phys. Status Solidi A 83, 159 (1984).

[3] B.V. Kobrin, V.P. Shantarovich, T.I. Kim, M.D. Michailov, Z.U. Borisova, J. Non-Cryst. Solids 89, 263 (1987).

[4] K.O. Jensen, P.S. Salmon, I.T. Penfold, P.G. Coleman, J. Non-Cryst. Solids 170, 57 (1994).

[5] M.J. Puska, C. Corbel, R.M. Nieminen, Phys. Rev. B 41, 9980 (1990).

[6] A. Feltz, Amorphe und Glasartige Anorganische Festkörper, Akademie-Verlag, Berlin 1983

[7] J. Kansy, Nucl. Instrum. Methods Phys. Res. A 374, 235 (1996). 
[8] R. Krause-Rehberg, H.S. Leipner, Positron Annihilation in Semiconductors. Defect Studies, Springer-Verlag, New York 1999.

[9] O. Shpotyuk, J. Filipecki, Free Volume in Vitreous Chalcogenide Semiconductors: Possibilities of Positron Annihilation Lifetime Study, WSP, Czestochowa 2003, p. 114.

[10] M.A. Popescu, J. Non-Cryst. Solids 35-36, 549 (1980).

[11] S.R. Elliott, Nature 354, 445 (1991).

[12] J. Bletry, Phylos. Magn. B 62, 469 (1990). 